\title{
The AKARI IRC asteroid flux catalogue: updated diameters and albedos ${ }^{\star}$
}

\author{
V. Alí-Lagoa ${ }^{1}$, T. G. Müller ${ }^{1}$, F. Usui ${ }^{2}$, and S. Hasegawa ${ }^{3}$ \\ ${ }^{1}$ Max-Planck-Institut für extraterrestrische Physik, Giessenbachstrasse 1, 85748 Garching, Germany \\ e-mail: vali@mpe.mpg.de \\ ${ }^{2}$ Center for Planetary Science, Graduate School of Science, Kobe University, 7-1-48 Minatojima-Minamimachi, Chuo-Ku, \\ Kobe 650-0047, Japan \\ ${ }^{3}$ Institute of Space and Astronautical Science, Japan Aerospace Exploration Agency, 3-1-1 Yoshinodai, Chuo-ku, \\ Sagamihara 252-5210, Japan
}

Received 21 August 2017 / Accepted 15 December 2017

\begin{abstract}
The AKARI IRC all-sky survey provided more than twenty thousand thermal infrared observations of over five thousand asteroids. Diameters and albedos were obtained by fitting an empirically calibrated version of the standard thermal model to these data. After the publication of the flux catalogue in October 2016, our aim here is to present the AKARI IRC all-sky survey data and discuss valuable scientific applications in the field of small body physical properties studies. As an example, we update the catalogue of asteroid diameters and albedos based on AKARI using the near-Earth asteroid thermal model (NEATM). We fit the NEATM to derive asteroid diameters and, whenever possible, infrared beaming parameters. We fit groups of observations taken for the same object at different epochs of the survey separately, so we compute more than one diameter for approximately half of the catalogue. We obtained a total of 8097 diameters and albedos for 5170 asteroids, and we fitted the beaming parameter for almost two thousand of them. When it was not possible to fit the beaming parameter, we used a straight line fit to our sample's beaming parameter-versus-phase angle plot to set the default value for each fit individually instead of using a single average value. Our diameters agree with stellar-occultationbased diameters well within the accuracy expected for the model. They also match the previous AKARI-based catalogue at phase angles lower than $50^{\circ}$, but we find a systematic deviation at higher phase angles, at which near-Earth and Mars-crossing asteroids were observed. The AKARI IRC All-sky survey is an essential source of information about asteroids, especially the large ones, since, it provides observations at different observation geometries, rotational coverages and aspect angles. For example, by comparing in more detail a few asteroids for which dimensions were derived from occultations, we discuss how the multiple observations per object may already provide three-dimensional information about elongated objects even based on an idealised model like the NEATM. Finally, we enumerate additional expected applications for more complex models, especially in combination with other catalogues.
\end{abstract}

Key words. minor planets, asteroids: general - catalogs

\section{Introduction}

Many asteroids are relatively intact fossils of the formation of the solar system and contain information about early dynamical phases. As representatives of the population of planetesimals that formed the terrestrial planets, they also provide constraints on planet formation and composition models. To gain insight into these topics, however, we also need to fully understand the evolutionary processes that asteroids have undergone such as collisions, differentiation, or space weathering, and what imprints these have left on their physical properties; for example, their size frequency distributions, shapes, rotational states, and surface physical properties (for a review, see Michel et al. 2015).

Asteroid diameters can be estimated by fitting models of their thermal emission to observations at thermal infrared wavelengths, at which said emission peaks as a consequence of their typical surface temperatures. This technique, called radiometry,

* Full Table 1 is only available at the CDS via anonymous ftp to cdsarc.u-strasbg.fr $(130.79 .128 .5)$ or via http://cdsarc.u-strasbg.fr/viz-bin/qcat? J/A+A/612/A85 has been the major contributor of asteroid diameters since the 1980s, when the Infrared Astronomical Satellite (IRAS) allowed two thousand asteroid diameters to be determined (Tedesco 1986; Tedesco et al. 2002b). After a modest addition of observations of approximately 150 more asteroids by the Midcourse Space Experiment (MSX) in the 1990s (Tedesco et al. 2002a), there has been two major space-based all-sky surveys at thermal infrared wavelengths in the last ten years: AKARI (Murakami et al. 2007) and WISE/NEOWISE (Wright et al. 2010; Mainzer et al. 2011a).

Usui et al. (2011), henceforth U11, used the 9- and 18- $\mu \mathrm{m}$ AKARI all-sky fluxes to produce the Asteroid catalogue using AKARI (AcuA), comprising 5000 asteroid diameters and visible geometric albedos based on an empirical calibration of the standard thermal model (STM) (see e.g. Lebofsky et al. 1986). After the release of the AcuA, the AKARI flux catalogue was updated with serendipitous asteroid detections among the IRC slow-scan observations (Takita et al. 2012), which were used by Hasegawa et al. (2013), H13, to add 88 completely new or updated diameters and visible geometric albedos. These works completed the catalogue of main-belt asteroids (MBA) down to diameters of $\sim 20 \mathrm{~km}$ (Usui et al. 2013, 2014). 
If AKARI doubled the IRAS catalogue in terms of numbers, WISE/NEOWISE increased the number by two orders of magnitude, providing observations of hundreds of near-Earth asteroids (NEAs; Mainzer et al. 2011a), more than a hundred thousand MBAs (Masiero et al. 2011), and thousands of outer MBAs and Jupiter Trojans (Grav et al. 2012). Still, as discussed by Usui et al. (2014), the AKARI and IRAS catalogues constitute important complements to NEOWISE at the large-size end of the distribution by compensating for the absence of a small but crucial number of large asteroids. In addition, the WISE detectors saturated with the largest targets, and although partial saturation corrections have been applied successfully (Mainzer et al. 2011b) to estimate diameters using a thermal model, they may not be optimal for more sophisticated thermo-physical models. Furthermore, the available catalogues also complement each other in that they provide coverage of different parts of the surfaces of many asteroids at different epochs and, especially those with more eccentric orbits, at different observation geometries. In this sense, AKARI is the longest-lasting fully-cryogenic survey with a duration of 18 months, whereas WISE/NEOWISE (WISE Cryogenic Survey) and IRAS spanned 8 and 9 months, respectively.

In this work, our aims are to provide the AKARI IRC flux catalogue to the community and to discuss several scientific applications to asteroid studies. As an example, we recompute asteroid diameters with the near-Earth asteroid thermal model (NEATM; Harris 1998) with an upgraded version of the implementation used in Alí-Lagoa \& Delbo' (2017). The NEATM has become the standard method in the field, so this new catalogue can facilitate comparison with others based on space IR surveys, like the one by Ryan \& Woodward (2010) from IRAS/MSX data, or Mainzer et al. (2016) based on NEOWISE. Our visible geometric albedos are computed from our new diameters and the IAU $H-G_{1}-G_{2}$ system (Muinonen et al. 2010). More specifically, we took the $H-G_{12}$ values provided by Oszkiewicz et al. (2011); this alternative is better suited for cases where the optical data coverage is not sufficient to reliably derive $G_{1}$ and $G_{2}$.

The structure of the article is as follows. In Sect. 2 we provide relevant information and references about the production of the flux catalogue (Sect. 2.1), our thermal model approach and its validation (Sects. 2.2 and 2.3), and we show a sample of our new diameters catalogue (Sect. 2.4). In Sect. 3 we compare our catalogue with the previous version and with WISE/NEOWISE diameters and albedos (Sects. 3.1 and 3.2), and we discuss several particular cases to illustrate the advantages and disadvantages of our approach in the context of the beaming parameter (Sect. 3.3). Finally, in Sect. 4, we conclude with some further expected scientific applications of the AKARI IRC All-sky Survey asteroid flux catalogue.

\section{Production of the catalogue}

\subsection{AKARI Infrared Camera All-sky Survey asteroid fluxes}

The AKARI IRC All-sky Survey asteroid flux catalogue has been publicly available in the JAXA website since October $2016^{1}$. Details about the mission concept (Murakami et al. 2007), the Infrared Camera (IRC; Onaka et al. 2007), point-source detection and calibration (Ishihara et al. 2010), and the production of the asteroid catalogue in particular were provided by Usui et al. (2011). Likewise, Hasegawa et al. (2013) explained the

1 http://www.ir.isas.jaxa.jp/AKARI/Archive/Catalogues/ Asteroid_Flux_V1/ procedures followed to obtain the 89 slow-scan detections of 88 MBAs. In total, we used 20773 observations.

The IRC asteroid flux catalogue includes data in bands $S 9 \mathrm{~W}$ and $L 18 W$, ranging from 6.7 to $11.6 \mu \mathrm{m}$ and from 13.9 to $25.6 \mu \mathrm{m}$, respectively. In addition to the measured fluxes, the catalogue contains the asteroid number, name, and/or provisional designation, the epoch, sky coordinates, and the geometry of observation, that is, the heliocentric distance $(r)$, geocentric distance $(\Delta)$, phase angle $(\alpha)$, the angle subtended by the Sun and the observer as seen from the asteroid, and the solar elongation which, by the spacecraft's design, is always within $1^{\circ}$ from $90^{\circ}$ (this configuration is called "quadrature").

It is necessary to colour-correct the reported fluxes because the spectral energy distributions (SEDs) of asteroids differ significantly over the widths of the two bandpasses from those of the K- and M-type giant stars used as calibrators. Colourcorrected fluxes and corresponding error bars are also given in the catalogue. They are based on a third-order polynomial fit (see Table 2 in Usui et al. 2011) to the colour corrections computed for a $10 \%$-albedo asteroid as a function of heliocentric distance. While the temperature at a given heliocentric distance can differ by a few $\mathrm{K}$ for very low- or high-albedo asteroids, we estimated that the corresponding colour corrections do not vary significantly to introduce a strong bias, especially for L18W data. For very-low-albedo Jupiter Trojans, however, this approximation could result in colour corrections for band S9W being underestimated by up to $4 \%$, so it may be advisable to recompute them for the purposes of thermo-physical modelling. The filter response functions (Onaka et al. 2007) are available at the JAXA website ${ }^{2}$. For our purposes here this is not so determinant, as fitting the beaming parameter can easily compensate for this small effect.

\subsection{Thermal modelling}

We used the near-Earth asteroid thermal model of Harris (1998; NEATM) as implemented in Alí-Lagoa \& Delbo' (2017), where the method is described in detail. In summary, the NEATM approximates the asteroid as a non-rotating sphere with idealised surface properties: it behaves as a grey body with constant emissivity of 0.9 and it does not conduct heat towards the subsurface, that is, each surface element reaches thermal equilibrium instantaneously so there is no thermal inertia (see Harris \& Lagerros 2002; Delbo et al. 2015, and references therein). To compensate for all simplifying assumptions and better fit thermal infrared data, the NEATM uses the infrared beaming parameter $\eta$ as a free parameter. It was empirically introduced in the framework of the standard thermal model to account for the effects of surface roughness and the tendency of rough surfaces to "beam" their thermal emission in the sunward direction (Lebofsky et al. 1986).

The NEATM was conceived to obtain more accurate diameters of near-Earth asteroids (NEAs) as the empirical phase factor calibrated from large main-belt asteroid observations was not appropriate for the typically high phase angles at which NEAs are observed. Since the fitted beaming parameter values correlate with the phase angle of the observation, $\alpha$ (Delbo' et al. 2003; Wolters \& Green 2009), it is customary to use a default value $\left(\eta_{d}\right)$ based on the average of particular asteroid populations whenever it is not possible to fit it (two or more thermal bands are required to have the necessary degree of freedom). An average value of

2 http://WWW.ir.isas.jaxa.jp/AKARI/Observation/ support/IRC/RSRF/ 
1.4 was found for NEAs (Mainzer et al. 2011a), 1.2 for Marscrossing asteroids (Alí-Lagoa \& Delbo' 2017), 1.0 for MBAs (Masiero et al. 2011), and 0.77 for Hildas and Jupiter Trojans (Grav et al. 2012). Here we considered our sample's statistics to choose a default value of $\eta$ as a function of $\alpha$. Figure 1 shows all our $\eta$-values based on two or more data points with $S / N>10$ in each band as a function of phase angle (green circles) and the corresponding best-fitting straight line, namely

$\eta_{d}(\alpha)=(0.009 \pm 0.001) \mathrm{deg}^{-1} \alpha+(0.76 \pm 0.03)$.

The figure also illustrates how taking data with $S / N>10$ only and requiring at least two measurements in each band removes the majority of extreme values of beaming parameter outside physical limits (usually set at $\sim 0.5$ and $\pi$ ). But since the IRC survey obtained typically two observations per sighting per object, which is not enough to gain good rotational coverage, it is still possible to obtain some high/low $\eta$-values when fitting data even with reasonable signal-to-noise ratios $(\mathrm{S} / \mathrm{N})(\mathrm{e}$.g. elongated objects observed when their visible projected area was minimum/maximum). To mitigate this, we did not include any fit with $\eta$-values outside the 5- $\sigma$ limits defined by the uncertainties of the coefficients in Eq. (1). Instead, we reran our model with a default value of $\eta$ taken from Eq. (1).

Arguably, fitting a straight line to the correlation between the NEATM beaming parameter and the phase angle of observation (Fig. 1) might not be the optimal option as the fit is not statistically robust. However, although the correlation has been reported and discussed several times in the literature (e.g. Delbo' et al. 2003; Wolters \& Green 2009; Masiero et al. 2011; Harris \& Drube 2016), it is still not well understood on purely physical grounds, likely because the beaming parameter is not a physical quantity. Thus, we consider it preferable to fit a straight line to our sample rather than any other function that may provide a statistically better fit. Furthermore, Eq. (1) reproduces diameters of objects in both extremes of the phase angle range satisfactorily and leads to default values of $\eta$ that are similar to those adopted for NEAs and Jupiter Trojans in NEOWISE works.

Regardless of whether $\eta$ was fitted or not, in the very few cases when our fits lead to unrealistic values of visible geometric albedos (we chose the limits to be $p_{V}>1.0$ or $p_{V}<0.025$ ), we reran the model increasing or decreasing the beaming parameter by $15 \%$. For instance, improving a fit that leads to an unrealistically low albedo requires a lower beaming parameter since this leads to a smaller diameter, which in turn would result in a higher value of $p_{V}$ given the $p_{V}-D-H$ relation:

$p_{V}=\left(\frac{D_{0}}{D} 10^{-H / 5}\right)^{2}$

where $H$ is the absolute magnitude and $D_{0}=1329 \mathrm{~km}$ (e.g. Pravec \& Harris 2007). We note that readjusting the beaming parameter based on an unrealistic value of albedo can be counterproductive if it is the $H$ value and not the thermal IR flux that is actually producing such an albedo value. However, given that we sometimes only have one IR flux to fit, we consider this approach more robust.

We used the $H-G_{12}$ values computed by Oszkiewicz et al. (2011), from now on referred to as O11. Vereš et al. (2015, hereafter V15) also produced a catalogue of $H-G_{12}$ values based on Pan-STARRS photometry and found systematic differences with respect to the Minor Planet Center and O11 absolute magnitudes but not with respect to those obtained by Pravec et al. (2012) based only on high-quality photometry. However, O11

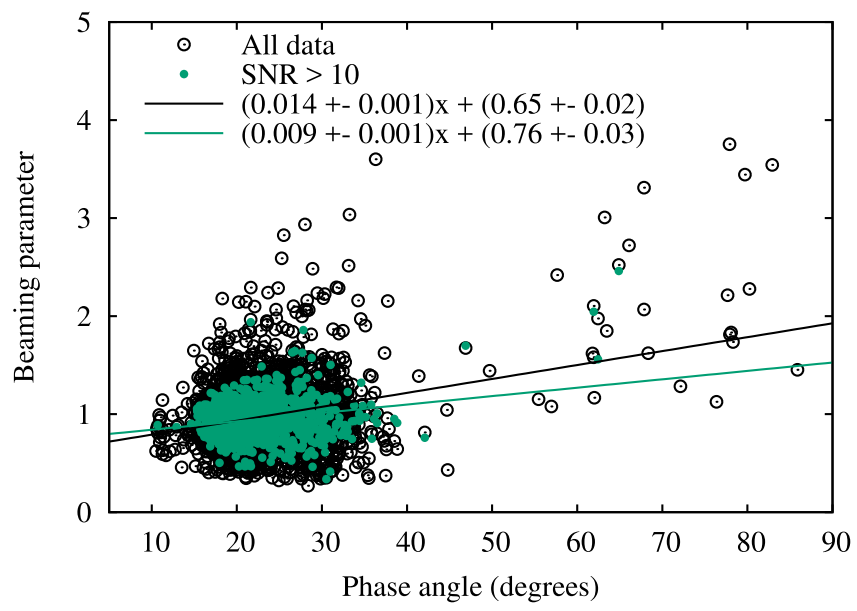

Fig. 1. Best-fitting beaming parameter values as a function of phase angle and corresponding best-fitting straight lines. Black circles: fits based on the complete catalogue ( $\approx 3000$ fits). Green circles: fits based on two or more data points in both AKARI bands with $S / N>10(\approx 1000$ fits).

and V15 have different strengths (see the discussion in V15), and a considerable fraction of objects in our catalogue are not included in the V15 catalogue. At any rate, it is possible to update the albedos in our catalogue with new $H$ values using Eq. (2), but making a detailed comparison is beyond the scope of this work.

\subsection{Validation}

To validate our model, in this section we compare the radiometric diameters of U11/H13, NEOWISE (Mainzer et al. 2016), and this work, with diameters derived from fits to stellar occultation chords compiled by Dunham et al. (2016). We computed the relative differences between each value of radiometric diameter and $a, b$ and $D_{\text {eq }}=\sqrt{a b}$, where $a$ and $b$ are the major and minor axes of the best-fitting ellipse and $D_{\text {eq }}$ is the diameter of the circle with the same area. For each entry, we took the minimum and the maximum differences and plotted them in Fig. 2, which shows the histograms we obtained for the diameters of U11H13 (A; it includes 184 objects), NEOWISE (B, 178 objects), and this work (C, 184 objects). The mean and average values of the minimum relative differences (empty boxes) are all close to zero and the standard deviations are of the order of $10 \%$, in consistency with minimum NEATM uncertainty estimates of other previous works (Harris 2006; Mainzer et al. 2011b).

The maximum relative differences are more widely spread $(\sigma \approx 30 \%)$ and deviate somewhat more systematically from zero, with averages of $\sim 6 \%$ for U11H13 and NEOWISE, and $\sim 10 \%$ for our diameters. This feature is not unexpected since radiometric diameters derived from high-quality thermal IR data are more likely to capture different cross-sections of irregular bodies as they rotate and thus provide a reliable volumeequivalent diameter. On the other hand, very accurate occultation chords are two-dimensional measures of size and, albeit "ground truth" estimates, they are more likely to lead to underestimates of the diameter and volume of very irregular bodies. In addition, the fact that the AKARI IRC survey provided fewer observations per sighting than WISE/NEOWISE could also explain why we obtain a higher average maximum deviation for our sample, as it does not guarantee an average of large and small cross-sections (see Sect. 3.3 for an example and further discussion). 


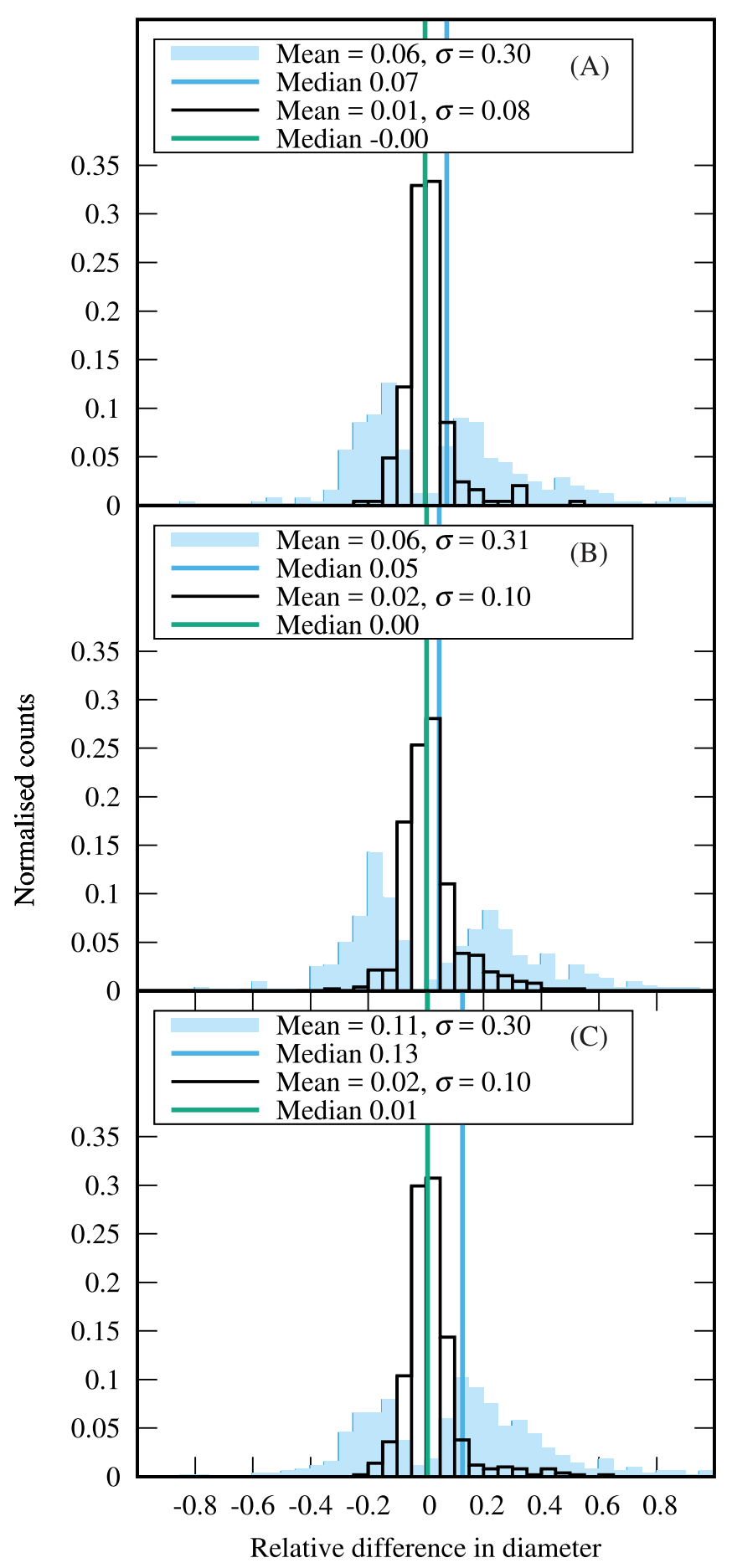

Fig. 2. Maximum (blue) and minimum (empty boxes) relative differences between radiometric sizes and occultation sizes, i.e. ( $D-$ $\left.x_{\text {Occ }}\right) / x_{\text {Occ }}$, where $x_{\text {Occ }}$ is either the long axis, the minor axis, or the equivalent diameter of the ellipse fitted to the occultation data. Panel A: Usui et al. (2011). Panel B: Mainzer \& NEOCam Science Team (2016). Panel $C$ : this work.

\subsection{The new diameter, albedo, and beaming parameter catalogue}

Table 1 shows a sample with a few lines of our new catalogue, which contains a total of 8097 fits for 5198 different bodies. Some asteroids have more than one entry in our new catalogue because the beaming parameter depends on the geometry of observation and requires that groups of observations of the same object taken at widely different epochs be fitted separately. Following (Mainzer et al. 2011b), we set three days as the maximum separation between consecutive data to be modelled together.

The diameters, albedos, and beaming parameters are provided with other relevant information, such as the $H-G_{12}$ values (Muinonen et al. 2010; Oszkiewicz et al. 2011), the number of data modelled in each band, the geometry of observation obtained from the Miriade ephemerides server ${ }^{3}$, and the epoch.

As in previous works, minimum error bars in $D$ are $10 \%$ $\left(20 \%\right.$ in $\left.p_{V}\right)$ if the beaming parameter was fitted, and $20 \%(40 \%$ in $p_{V}$ ) otherwise. Diameter fits to single data points are provided for completeness but should be taken with caution.

\section{Discussion}

\subsection{Comparison with $\mathrm{U} 11 / \mathrm{H} 13$}

In Sect. 2.3 we showed that we obtain statistically equivalent diameters when we compare with occultation-based cross sections. But the differences between our approach and that of U11/H13 become systematically larger for objects observed at high phase angles, that is, NEAs and some Mars-crossing asteroids. This is illustrated in Fig. 3, where we plot the relative difference in diameter as a function of phase angle for objects with fitted $\eta$ (top panel) and with our calibrated default beaming parameter function, $\eta_{d}(\alpha)$ (lower panel). The colour code, related to the density of points in the plot, shows that a large fraction of cases at phase angles below $40^{\circ}$ are well within the expected $\pm 10 \%$ relative differences, whereas the NEATM approach leads to systematically larger diameters in the range $50^{\circ}<\alpha<90^{\circ}$, in creasing from $10 \%$ to $20 \%$. An example is the potentially hazardous NEA (7341), for which U11 reported $D=0.78 \mathrm{~km}$ and $p_{V}=0.62$; instead, we found $D=1.2 \mathrm{~km}$ and $1.1 \mathrm{~km}$, and the corresponding $p_{V}=0.23$ and 0.31 derived for very similar $H$-values. Our albedos are more compatible with the spectral classification of Sq found for this object (Bus \& Binzel 2002a,b).

Figure 3 also shows a small component of objects at intermediate phase angles for which we obtain significantly different diameters. These are a consequence of a disadvantage in our approach: fitting groups of data separately means that sometimes we are left with one or two observations, often in a single band, which can lead to inaccurate diameters. The reason not to average these diameters with other values in our catalogue obtained for the same objects is, as pointed out in Sects. 2.2 and 3.3, to preserve potentially useful information about irregular objects. In addition, it is not useful to average an inaccurate diameter with a more reliable value.

From Eq. (2), the differences in albedo are a factor of two of the differences in diameter if the $H$ value does not change. But many absolute magnitudes have indeed been updated since U11 and H13, some by up to one magnitude. Because the temperatures are proportional to $(1-A)^{\frac{1}{4}}$ and the asteroids' Bond albedos $A$ are typically $<0.4$, the impact of the $H$-values on our diameters is small (see Sect. 3 in Alí-Lagoa \& Delbo' 2017). We show this in Fig. 4, where the first two panels show how the relative differences between the diameters of U11/H13 and ours are not strongly correlated to the differences in $H$ values, even when we are not able to fit $\eta$. The red and green areas, representing the large majority of our sample, lie within the -0.30 to +0.30 bounds of the expected 2- $\sigma$ uncertainties. This is not the case for the visible geometric albedos, however. The computed $p_{V}$ is a non-linear function of $H$, so we have an asymmetric trend in

3 http://vo.imcce.fr/webservices/miriade/ 
Table 1. Derived diameters $(D)$, visible geometric albedos $\left(p_{V}\right)$, and beaming parameters $(\eta)$.

\begin{tabular}{ccccccccccccc}
\hline \hline Object & $H$ & $G_{12}$ & $D(\mathrm{~km})$ & $p_{V}$ & $\eta$ & Fit & $\mathrm{n}_{9}$ & $\mathrm{n}_{18}$ & $r(\mathrm{au})$ & $\Delta(\mathrm{au})$ & $\alpha\left(^{\circ}\right)$ & $\mathrm{MJD}$ \\
\hline 00001 & 3.43 & 0.88 & 1082.6 & 0.064 & 1.05 & True & 2 & 1 & 2.95137 & 2.77621 & 20.01867 & 53868.7602 \\
00001 & 3.43 & 0.88 & 929.9 & 0.087 & 0.94 & False & 0 & 2 & 2.98621 & 2.82770 & 19.37133 & 54048.8055 \\
00001 & 3.43 & 0.88 & 1029.9 & 0.071 & 0.96 & False & 1 & 1 & 2.88090 & 2.69998 & 20.59184 & 54324.6499 \\
00002 & 4.22 & 0.68 & 556.99 & 0.117 & 1.00 & True & 3 & 3 & 3.38096 & 3.23953 & 17.24352 & 54006.1010 \\
00002 & 4.22 & 0.68 & 535.46 & 0.126 & 0.99 & True & 3 & 3 & 3.34383 & 3.18900 & 17.66837 & 54259.5766 \\
00003 & 5.19 & 0.18 & 268.28 & 0.206 & 1.14 & True & 3 & 2 & 2.96530 & 2.79400 & 19.37168 & 54116.1894 \\
00003 & 5.19 & 0.18 & 247.44 & 0.242 & 1.08 & True & 1 & 2 & 3.25764 & 3.10484 & 18.18754 & 54288.8980 \\
00004 & 2.99 & 0.36 & 562.61 & 0.355 & 1.10 & True & 2 & 3 & 2.18592 & 1.95199 & 26.91148 & 54154.8810 \\
00005 & 6.84 & 0.30 & 105.40 & 0.292 & 0.83 & True & 2 & 2 & 2.80221 & 2.61512 & 21.24749 & 53942.5128 \\
00005 & 6.84 & 0.30 & 104.53 & 0.297 & 0.84 & True & 2 & 1 & 2.45474 & 2.25927 & 23.63201 & 54119.4646 \\
\hline
\end{tabular}

Notes. The full version of the table is available in electronic form at the CDS. The albedos were computed from the absolute asteroid magnitudes $H$ taken from Oszkiewicz et al. (2011). Entries with "True" in the "Fit" column indicate that the beaming parameter was fitted; otherwise, the value was computed from Eq. (1). On the other hand, the diameters were always fitted. Columns $\mathrm{n}_{9}$ and $\mathrm{n}_{18}$ refer to the number of observations fitted in the 9- $\mu \mathrm{m}$ and 18- $\mu$ bands, and $r, \Delta$, and $\alpha$ are the heliocentric and geocentric distances and phase angle, respectively. MJD is the modified Julian date of the last observation of the group. Typical minimum uncertainties are higher when the beaming parameter could not be fitted, as discussed in the main text.

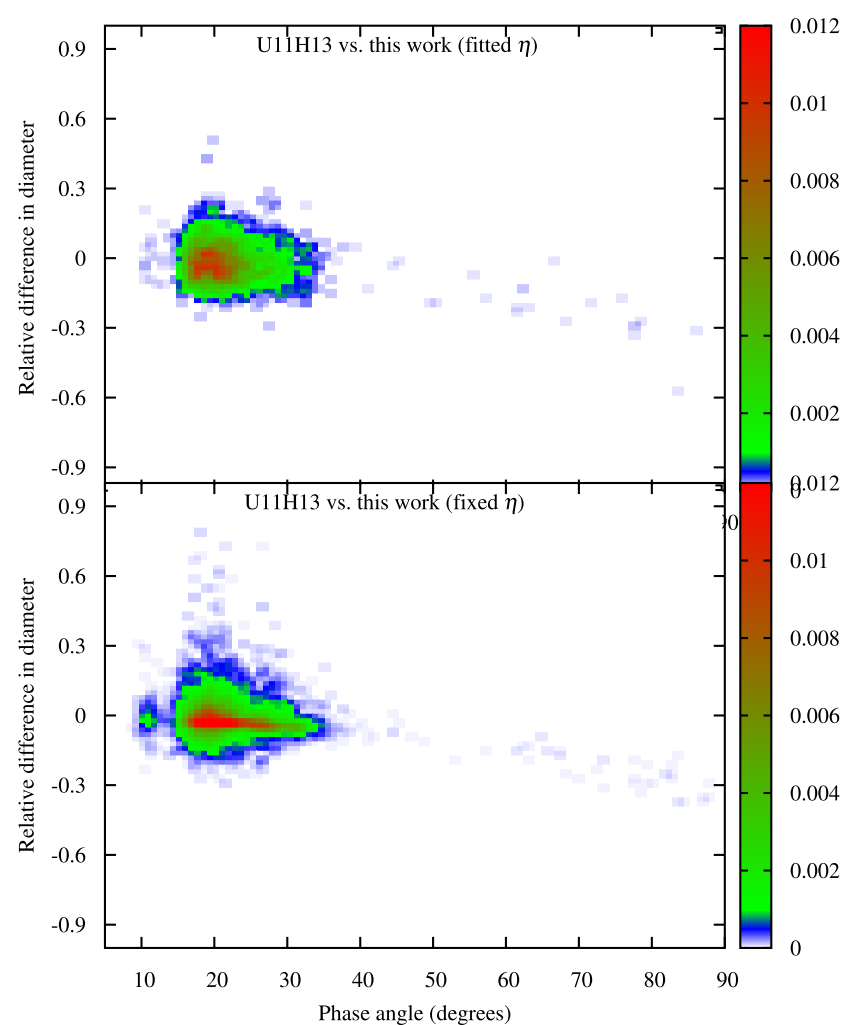

Fig. 3. Relative differences between the diameters of U11/H13 and ours as a function of phase angle. The colour map indicates the number density of points in the box normalised to the total number of points in the plot. Top: cases for which $\eta$ could be fitted. Bottom: $\eta_{d}$ was taken as default.

the lower panels of the figure (we highlight the change in scale of the $y$-axis). For a small percentage of the sample the albedos have halved and some have even decreased by a factor of 3 . This resulted in an improvement in those cases when U11/H13 reported unrealistically high values of $p_{V}$, such as (840), (1600), or (3873), for example. On the other hand, we also obtained some unrealistically low $p_{V}$-values $(<0.025)$. These were often based on fits to a single data point, so we iteratively reran our model increasing the $\eta_{d}$-value by $10 \%$ until a more physical value of albedo was obtained.

\subsection{Intercomparison with WISE/NEOWISE diameters and albedos}

Figure 5 shows density maps for plots of albedo versus diameter for the whole sample computed here, the values of U11H13, and NEOWISE. We find the two major albedo groups expected for the compositionally heterogeneous asteroid population and very similar $p_{V}$-versus- $D$ plots when compared to U11 and NEOWISE (for a comparative study of MBA albedo statistics based on AKARI and WISE/NEOWISE thermal models see Usui et al. 2014). We find two differences worth mentioning. The intermediate albedo cluster $\left(0.10<p_{V}<0.20\right)$ likely comprising X-complex asteroids (DeMeo et al. 2009; Mainzer et al. 2011c) seems to be more separated from the low-albedo cloud in both our sample and the NEOWISE catalogue, whereas the U11H13 low-albedo cloud extends a little towards $p_{V}>0.10$. This could be partly related to the update of many $H$ values for asteroids in the $10-30 \mathrm{~km}$ range after the U11 work was completed. The second difference is that the NEOWISE clusters stretch towards higher $p_{V}$ at lower sizes, which results in a negative slope that is not apparent in the AKARI catalogues. This could be partly due to the contribution of diameters derived from data taken during non-cryogenic phases of the mission and the default values of beaming parameter assumed, namely 1.0 (Masiero et al. 2012) and 0.95 (Nugent et al. 2015, 2016). Those values are more representative of medium-sized and large objects $(10 \mathrm{~km}<D<100 \mathrm{~km})$, whereas smaller bodies tend to require higher beaming parameters (all other variables of the model being equal; see the discussions by Alí-Lagoa et al. 2016; Alí-Lagoa \& Delbo' 2017). Because underestimating $\eta$ results in a smaller diameter, it leads to a higher albedo.

\subsection{Infrared beaming parameters}

Table 2 includes a sample of diameter fits that we obtained for several objects compared to values taken from U11, the NEOWISE catalogue, occultations, or adaptive optics. These examples show how the different diameter fits obtained for the same object based on separate groups of observations (see 
Table 2. Sizes of several asteroids based on radiometry from this work, U11/H13, and NEOWISE ( $D_{\mathrm{W}}$; Mainzer et al. 2016), and those determined from ellipsoids fitted to stellar occultation chords (compiled by Dunham et al. 2016), and based on adaptive optics (AO; Hanuš et al. 2017a,b).

\begin{tabular}{lccccccc}
\hline \hline Asteroid & $D(\mathrm{~km})$ & $D_{\mathrm{U} 11}(\mathrm{~km})$ & $D_{\mathrm{W}}(\mathrm{km})$ & Occ. $a \times b(\mathrm{~km})$ & $D_{\text {Occ,eq }}(\mathrm{km})$ & $\mathrm{AO} a \times b \times c(\mathrm{~km})$ & $D_{\mathrm{AO}, \mathrm{eq}}(\mathrm{km})$ \\
\hline$(9)$ & $\mathbf{2 2 3}, 160$ & 166 & 183,184 & $176 \times 161$ & 168 & - & 168 \\
& & & & $200 \times 137$ & 166 & & \\
& & & & $182 \times 153$ & 167 & & \\
$(10)$ & 454,403 & 428 & $\mathbf{5 3 3}$ & $464 \times 383$ & 422 & - & - \\
$(16)$ & $\mathbf{2 0 0}, 236$ & 207 & $\mathbf{2 8 8}$ & $235 \times 230$ & 233 & - & 225 \\
$(130)$ & 212,212 & 183 & $181, \mathbf{1 6 2 , 1 5 9}$ & $255 \times 154$ & 199 & $262 \times 205 \times 164$ & 206 \\
$(303)$ & 107,89 & 99,105 & $104, \mathbf{1 2 5}$ & $87 \times 110$ & 98 & - & - \\
$(1263)$ & 50,44 & 51 & $\mathbf{4 0 . 2 , 3 7 . 5 6}$ & $53.9 \times 36.2$ & 44 & - & - \\
& & & & & & & - \\
\hline
\end{tabular}

Notes. Equivalent diameters from occultation and AO fits are also shown. The numbers in bold face indicate that the values of the beaming parameter for those fits were fixed to a default value. If several radiometric values are available, we have kept them in the same column, separated by commas.

Sect. 2.2) are still compatible with the dimensions derived from fits to stellar occultation data. This means that high-quality AKARI data may be useful to identify irregular objects observed more than once if they were observed at widely different aspects; we have not averaged the diameter fits for this reason.

The case of (9) Metis is particularly interesting because it has also several recorded occultation events with high-quality chords. AKARI observed Metis on two occasions during the all-sky survey, so we derived two values for its diameter that differ by $\approx 20 \%$. Compared to the high-quality occultation fits 4 by Dunham et al. (2016), it would be tempting to reject our first diameter $(223 \pm 40 \mathrm{~km})$ over the second one $(160 \pm 20 \mathrm{~km})$. However, we took the convex shape model of (9) Metis available in the DAMIT database (Ďrech et al. 2010, 2011) and rendered $\mathrm{it}^{5}$ as an observer would have seen it at the different epochs. Figure 6 shows that the cross-sectional area of the object at epoch 1 , close to pole-on, was larger than in the second epoch. We also checked that the three occultation fits included in Table 2 were taken at more edge-on views ${ }^{6}$.

We also collected some other examples in Table 2 that show how choosing an inappropriate default value of $\eta$ may bias the radiometric diameter. The diameters of (10) Hygiea and (16) Psyche given by Mainzer et al. (2016) were based on a default beaming parameter of 1.20 and 1.0, respectively, whereas large main-belt asteroids' thermal data tend to be better fitted by $\eta$ values in the range 0.7-0.9 (e.g. Masiero et al. 2014; Alí-Lagoa et al. 2016). Likely as a consequence, the corresponding diameters are in both cases larger than those found from occultations and adaptive optics.

Conversely, because AKARI did not sample each object with as many observations per sighting as WISE/NEOWISE (cf. from one to five data per band versus an average of ten), some lowerquality AKARI fluxes occasionally lead to unrealistic $\eta$ fits (as

4 Quality codes 3 and 4.

5 We used the ISAM service: http://isam.astro.amu.edu.pl (Marciniak et al. 2012).

6 Incidentally, the only occultation event taken at a view closer to poleon does present a larger cross section, but we did not include it in our table because it was assigned a quality code 2 by Dunham et al. (2016). those apparent in Fig. 1) and correspondingly inaccurate diameters and albedos. Thus, we do not expect this projected area effect to satisfactorily explain all differences between radiometric diameters obtained from different epochs of observations by AKARI or indeed any other survey. Given the current visualisation tools that we have at our disposal, we cannot examine all hundreds of cases individually, so we reiterate that these relative differences should be taken as representative of the minimum error in diameter. In this sense, because NEOWISE took more observations per sighting per object, it samples the rotational phases better and is thus less prone to this problem than AKARI. However, some degree of bias is still unavoidable, especially for irregular objects that were observed only once (single apparition) at thermal IR wavelengths. This effect will be even stronger on the values of the albedo (Eq. (2)), since the $H$ magnitudes are (usually) based on several apparitions.

\section{Outlook and concluding remarks}

We provide the thermal infrared fluxes obtained for over 5000 asteroids by the AKARI IRC All-sky survey, and an updated catalogue of diameters and albedos based on the NEATM, which allowed us to fit the beaming parameter in numerous cases. The visible geometric albedos were computed from the diameters and the tabulated IAU $H-G_{12}$ parameters (Oszkiewicz et al. 2011). We validated our approach by comparing our diameters with dimensions obtained from fits to stellar occultation chords of over 100 asteroids, and we discussed the usefulness of the catalogue by comparing its global properties against the AcuA (U11,H13) and NEOWISE catalogues (Sects. 3.1 and 3.2).

In Sect. 3.3 we focused on a few examples that illustrate how AKARI IRC fluxes may provide three-dimensional information of elongated/irregular asteroids based on the two or three groups of observations it recorded. Although this is the main reason why we have not averaged our multiple diameters, there are also some cases in which there were only one or two measurements, often in one single band, so we argue that it is not advantageous to average less accurate estimates with more reliable ones. By the same token, we did not average with other diameters derived 


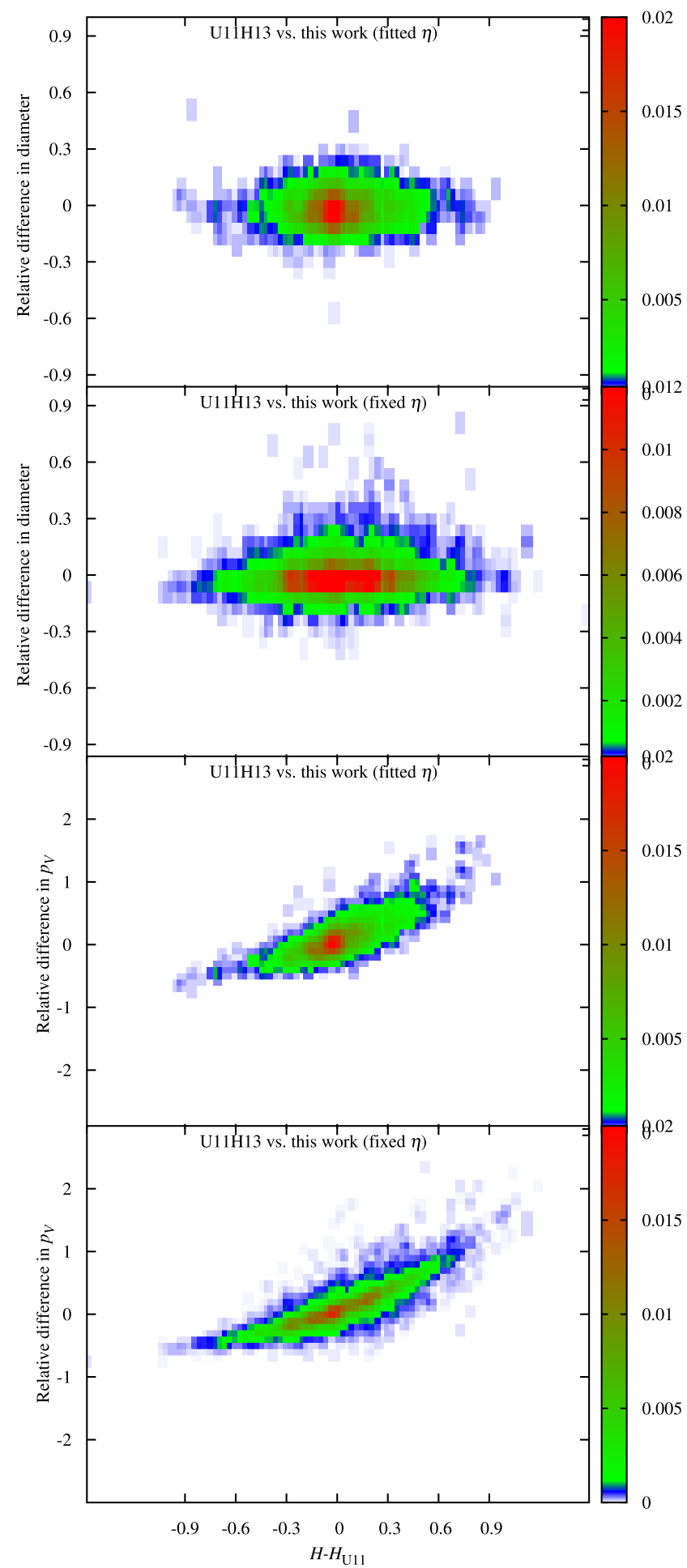

Fig. 4. Relative differences between the diameters of U11/H13 and ours (top panels) as a function of $H-H_{\mathrm{U} 11}$. The colour map indicates the number density of points in the box normalised to the total number of points in the plot. The bottom panels show the same plot but for the visible geometric albedos.

from WISE or IRAS because we have not yet accumulated the minimum of ten or fifteen estimates per object based on purely thermal data that would be required to obtain a statistically robust result. To fulfill this aim, more all-sky surveys would be ideal (e.g. Mainzer \& NEOCam Science Team 2016).

We emphasise two strengths of the AKARI catalogue versus the WISE/NEOWISE full cryogenic survey, which is by far the
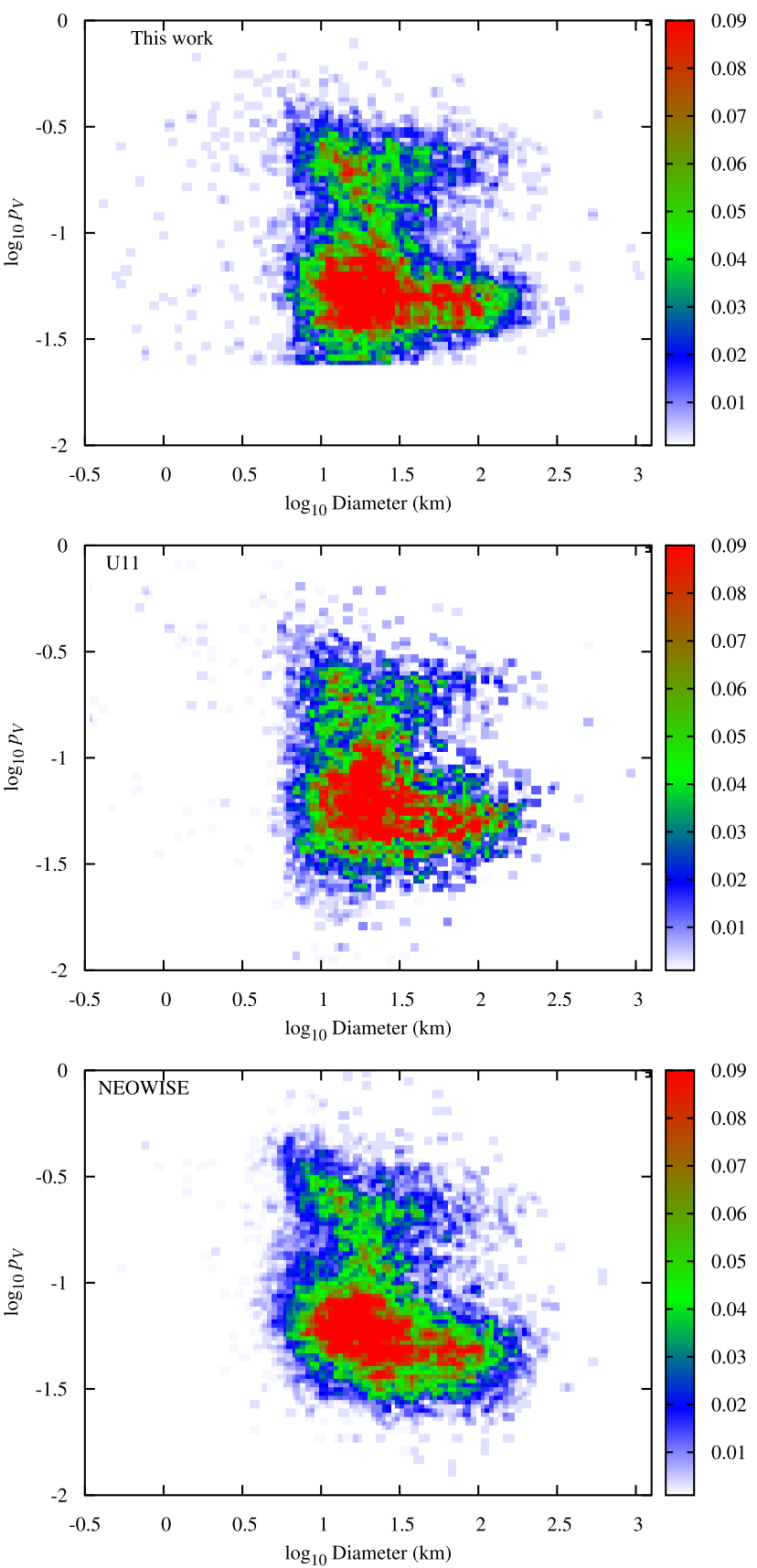

Fig. 5. Visible geometric albedos versus diameters derived here from the AKARI IRC All-sky survey fluxes (top panel), those of U11H13 (middle panel), and those computed for all our objects that were reported in the tables by Mainzer et al. (2016). The colour code is proportional to the number of points in each bin normalised to the total number of points, which is different in each case.

largest source of asteroid thermal IR data (see the comparative study by Usui et al. 2014): the AKARI detectors did not partially saturate for the largest targets, so AKARI is complete for mainbelt asteroids down to sizes of $20 \mathrm{~km}$ (Usui et al. 2013, 2014); the all-sky survey lasted roughly 18 months, covering more than $95 \%$ of the sky twice (cf. $\sim 8$ months of the WISE Cryogenic Survey, covering $\sim 20 \%$ twice). Specifically, 2000 asteroids in the catalogue were observed twice, and $\sim 200$ three times. From these, we can anticipate further scientific potential for more sophisticated thermo-physical modelling based on the combination of AKARI, WISE/NEOWISE, IRAS, Spitzer, Herschel and other sources (e.g. Mainzer et al. 2015). For example, we 


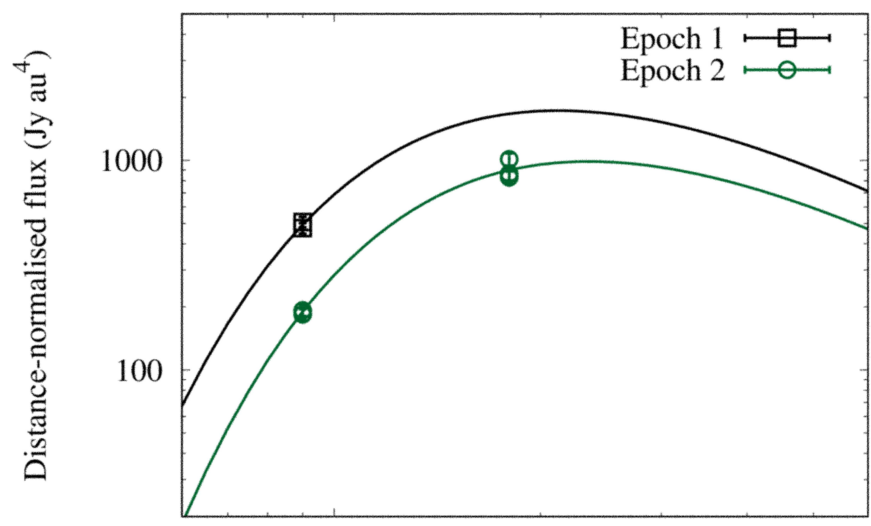

10

Wavelength $[\mu \mathrm{m}]$
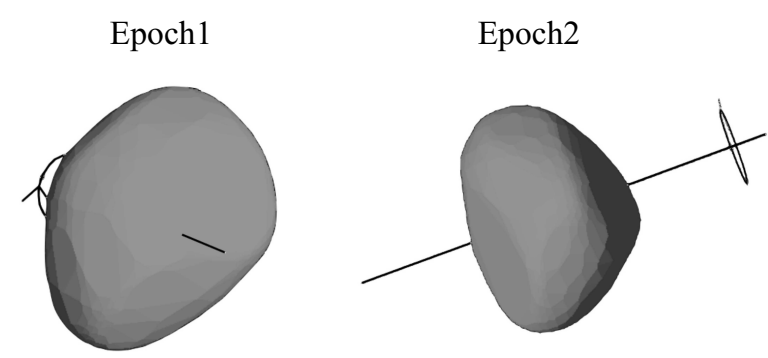

Fig. 6. Orientation of the convex model of (9) Metis (Torppa et al. 2003; Durech et al. 2011) at the two epochs at which it was observed by AKARI. The almost pole-on view (Epoch 1) resulted in a larger observed projected area compared to the edge-on one (Epoch 2). This is contributing significantly to the $20 \%$ discrepancy between the two diameters.

are starting to gather enough thermal IR observations before and after opposition for some key objects, which would be especially helpful to constrain surface thermo-physical properties whenever three-dimensional shape and spin axis orientation are available. For those without shape information, it could be possible to constrain the sense of rotation (Müller 2002; MacLennan \& Emery 2013). Also, some bodies in highly eccentric orbits have been observed at different heliocentric distances, which can open the possibility to study thermal inertia as a function of temperature. Ultimately, more accurate diameters are crucial to obtain accurate densities for the asteroids whose masses will be estimated thanks to Gaia (Tanga \& Mignard 2012), because the error in the diameter spreads threefold onto the volume estimate and thus dominates the error in the density, as pointed out by Carry (2012).

All these aspects are the focus of the "Small Bodies: Near And Far" (SBNAF), a project funded by the EU to carry out a benchmark study on small-body physical and thermal properties (three-dimensional shapes, rotational states, thermal inertias, etc.) to assess our current models' state of the art and to ensure a fruitful scientific exploitation of space- and ground-based data on which these models are based (Müller et al. 2017). In a broader context, accurate knowledge of the physical properties of large main-belt asteroids is extremely valuable for calibration purposes for ALMA and other millimetre and sub-millimetre observatories (Müller et al. 2014).

Acknowledgements. The research leading to these results has received funding from the European Union's Horizon 2020 Research and Innovation Programme, under Grant Agreement no 687378. This research is based on observations with AKARI, a JAXA project with the participation of ESA. The work of S.H. was supported by the JSPS KAKENHI Grant Numbers JP15K05277 and JP17K05636, and by the Hypervelocity Impact Facility (former facility name: the Space Plasma Laboratory), ISAS, JAXA. This research has made use of data and/or services provided by the International Astronomical Union's Minor Planet Center. This publication makes use of data products from NEOWISE, which is a project of the Jet Propulsion Laboratory/California Institute of Technology, funded by the Planetary Science Division of the National Aeronautics and Space Administration. This research also made use of the NASA/ IPAC Infrared Science Archive, which is operated by the Jet Propulsion Laboratory, California Institute of Technology, under contract with the National Aeronautics and Space Administration. This research has made use of IMCCE's Miriade VO tool. The thermal model code was based on a development of M. Delbo's implementation (Delbo' \& Harris 2002).

\section{References}

Alí-Lagoa, V., \& Delbo', M. 2017, A\&A, 603, A55

Alí-Lagoa, V., Licandro, J., Gil-Hutton, R., et al. 2016, A\&A, 591, A14

Bus, S. J., \& Binzel, R. P. 2002a, Icarus, 158, 146

Bus, S. J., \& Binzel, R. P. 2002b, Icarus, 158, 106

Carry, B. 2012, Planet. Space Sci., 73, 98

Delbo', M., \& Harris, A. W. 2002, Meteor. Planet. Sci., 37, 1929

Delbo', M., Harris, A. W., Binzel, R. P., Pravec, P., \& Davies, J. K. 2003, Icarus, 166,116

Delbo, M., Mueller, M., Emery, J. P., Rozitis, B., \& Capria, M. T. 2015, Asteroid Thermophysical Modeling, eds., P. Michel, F. E. DeMeo, \& W. F. Bottke, 107 DeMeo, F. E., Binzel, R. P., Slivan, S. M., \& Bus, S. J. 2009, Icarus, 202, 160

Dunham, D. W., Herald, D., Frappa, E., et al. 2016, NASA Planetary Data System, 243

Durech, J., Sidorin, V., \& Kaasalainen, M. 2010, A\&A, 513, A46

Durech, J., Kaasalainen, M., Herald, D., et al. 2011, Icarus, 214, 652

Grav, T., Mainzer, A. K., Bauer, J., et al. 2012, ApJ, 744, 197

Hanuš, J., Viikinkoski, M., Marchis, F., et al. 2017a, A\&A, 601, A114

Hanuš, J., Marchis, F., Viikinkoski, M., Yang, B., \& Kaasalainen, M. 2017b, A\&A, 599, A36

Harris, A. W. 1998, Icarus, 131, 291

Harris, A. W. 2006, in IAU Symposium, eds., L. Daniela, M. Sylvio Ferraz, \& F. J. Angel, Asteroids, Comets, Meteors, 229, 449

Harris, A. W., \& Drube, L. 2016, ApJ, 832, 127

Harris, A. W., \& Lagerros, J. S. V. 2002, Asteroids III (University of Arizona Press), 205

Hasegawa, S., Müller, T. G., Kuroda, D., Takita, S., \& Usui, F. 2013, PASJ, 65, 34

Ishihara, D., Onaka, T., Kataza, H., et al. 2010, A\&A, 514, A1

Lebofsky, L. A., Sykes, M. V., Tedesco, E. F., et al. 1986, Icarus, 68, 239

MacLennan, E. M., \& Emery, J. P. 2013, in AAS/Division for Planetary Sciences Meeting Abstracts, 45, 208.19

Mainzer, A., Grav, T., Bauer, J., et al. 2011a, ApJ, 743, 156

Mainzer, A., Grav, T., Masiero, J., et al. 2011b, ApJ, 736, 100

Mainzer, A., Grav, T., Masiero, J., et al. 2011c, ApJ, 741, 90

Mainzer, A., Usui, F., \& Trilling, D. E. 2015, Space-Based Thermal Infrared Studies of Asteroids, eds., P. Michel, F. E. DeMeo, \& W. F. Bottke, 89

Mainzer, A. K., \& NEOCam Science Team. 2016, in AAS/Division for Planetary Sciences Meeting Abstracts, 48, 327.01

Mainzer, A. K., Bauer, J. M., Cutri, R. M., et al. 2016, NASA Planetary Data System, 247

Marciniak, A., Bartczak, P., Santana-Ros, T., et al. 2012, A\&A, 545, A131

Masiero, J. R., Mainzer, A. K., Grav, T., et al. 2011, ApJ, 741, 68

Masiero, J. R., Mainzer, A. K., Grav, T., et al. 2012, ApJ, 759, L8

Masiero, J. R., Grav, T., Mainzer, A. K., et al. 2014, ApJ, 791, 121

Michel, P., DeMeo, F. E., \& Bottke, W. F. 2015, Asteroids IV (University of Arizona Press)

Muinonen, K., Belskaya, I. N., Cellino, A., et al. 2010, Icarus, 209, 542

Müller, T. G. 2002, Meteor. Planet. Sci., 37, 1919

Müller, T., Balog, Z., Nielbock, M., et al. 2014, Exp. Astron., 37, 253

Müller, T. G., Marciniak, A., Kiss, C., et al. 2017, Adv. Space Res., submitted, [arXiv: 1710.09161]

Murakami, H., Baba, H., Barthel, P., et al. 2007, PASJ, 59, S369

Nugent, C. R., Mainzer, A., Masiero, J., et al. 2015, ApJ, 814, 117

Nugent, C. R., Mainzer, A., Bauer, J., et al. 2016, AJ, 152, 63

Onaka, T., Matsuhara, H., Wada, T., et al. 2007, PASJ, 59, S401

Oszkiewicz, D. A., Muinonen, K., Bowell, E., et al. 2011, J. Quant. Spec. Radiat. Transf., 112, 1919

Pravec, P., \& Harris, A. W. 2007, Icarus, 190, 250

Pravec, P., Harris, A. W., Kušnirák, P., Galád, A., \& Hornoch, K. 2012, Icarus, 221,365 
V. Alí-Lagoa et al.: AKARI IRC: updated diameters and albedos

Ryan, E. L., \& Woodward, C. E. 2010, AJ, 140, 933

Takita, S., Ikeda, N., Kitamura, Y., et al. 2012, PASJ, 64, 126

Tanga, P., \& Mignard, F. 2012, Planet. Space Sci., 73, 5

Tedesco, E. F. 1986, in Asteroids, Comets, Meteors II, eds., C.-I. Lagerkvist,

H. Rickman, B. A. Lindblad, \& H. Lundstedt, 13

Tedesco, E. F., Egan, M. P., \& Price, S. D. 2002a, AJ, 124, 583

Tedesco, E. F., Noah, P. V., Noah, M., \& Price, S. D., 2002b, AJ, 123, 1056

Torppa, J., Kaasalainen, M., Michałowski, T., et al. 2003, Icarus, 164, 346
Usui, F., Kuroda, D., Müller, T. G., et al. 2011, PASJ, 63, 1117

Usui, F., Kasuga, T., Hasegawa, S., et al. 2013, ApJ, 762, 56

Usui, F., Hasegawa, S., Ishiguro, M., Müller, T. G., \& Ootsubo, T. 2014, PASJ, 66,56

Vereš, P., Jedicke, R., Fitzsimmons, A., et al. 2015, Icarus, 261, 34

Wolters, S. D., \& Green, S. F. 2009, MNRAS, 400, 204

Wright, E. L., Eisenhardt, P. R. M., Mainzer, A. K., et al. 2010, AJ, 140, 1868 